\title{
Antinutritional Factors And Mycotoxins As Natural Hazards Threaten Food Safety
}

\author{
Saad, M.M. \\ Dept. of Food Safety \& Contamination, NRC, Egypt
}

\begin{abstract}
Plants in general, edible plants and legumes in particular commonly synthesized a range of secondary metabolites as part of their protection against attack by herbivores, insects and pathogens. Such secondary metabolites identified as antinutritional factors (ANF's) and naturally occurred as means to survive in adverse growing conditions. The term antinutritional refers to defense metabolites having specific biological effects depending upon the structure of specific compounds which include proteins, glycosides, terpenoids and alkaloids. So far, it's proved that legumes are very rich source of antinutritional factors. It's worthy to state that if human or animals consume plants containing ANF's may exposed to adverse effects, but not lethal ones. Legumes are unique human food, because their rich nutrient content including carbohydrates and high ratio of proteins. Their nutritional content contributes to many health benefits to human. So, the knowledge regards various ANF's present in food represent the corner stone to control, minimize and reduce them in the diet. As well, the recent studies showed that low concentrations of the biological active ANF's proved to have positive health effects on both human and animal health. Simultaneously, mycotoxins which are also secondary metabolites naturally occurred on a wide range of plants when infected by certain species of fungi. Mycotoxins prevalence in food of plant origin may be relatively higher during post-harvest stages. During harvesting, handling, storage and distribution, agricultural commodities are subjected to invade and infect by toxigenic fungi, which are capable to yield the secondary metabolites of mycotoxins. This review article aims to focus on the necessary knowledge about the two categories of the secondary metabolites of ANF's and mycotoxins to assess researchers and food processors to maximize the positive effects of ANF's and to minimize or eliminate the risk of mycotoxins.
\end{abstract}

Keywords: Antinutritional factors - allelochemicals - mycotoxins - secondary metabolites.

\section{Introduction}

Recently, it's well established to consider antinutritional factors as anti-nutrients with negative effects and/ or nutritive compounds with positive effects on human health (Gemede and Ratta, 2014). Most of these ANF's are present in food of plant origin. Thus, the presence of cyanogenic glycosides and other ANF's may induce dramatic effects if their consumption exceeds certain limits. As well, certain harmful effects might also be due to the degraded products of ANF's. In fact, plants contain thousands of compounds which depending upon the situations could have beneficial and / or deleterious effects on organisms consuming them. ANF's may be regarded as a class of "allellochemicals" which are generally not lethal. They diminish metabolic pathways, but may also cause toxicity when the food rich in these active compounds is consumed in large quantities (FAO, 1979). The term allelochemials refer to toxic chemicals produced by plants to defend itself against herbivores and/ or competing organisms (Rocio and Ana, 2007). So, the released allellochemicals had negative effects on human and animal. Being an antinutritional factor is not an intrinsic characteristic of compounds, but depends upon the digestive process of the ingesting biological system. Trypsin inhibitors, as an example, do not exert adverse effects in ruminants because they are degraded in the rumen (Gemede and Ratta , 2014). Allelochemials, also known as inherent silent tool of self protection. The potential of these chemicals becoming recent bioactive chemicals useful for more sustainable agriculture and safe food production for human have been realized for quite some time (Fujii and Hiradate, 2007). The allelopathy hypothesis describes the real meaning of these secondary metabolites as a tool of immobile plants to protect themselves from surrounding other life that might attack them, or a tool to communicate each other life for survival. It has been commonly assumed that there are more than 500.000 plant species and more than 30.000 secondary natural chemical are available in our world (Fujii and Hiradote, 2007). The term allelopathy means an interaction between plant and each of, other plants, insects, microorganisms and animals through bioactive natural compounds. A related concept of antibiotics which is an action of natural chemicals produced by microorganisms to other biota.

Agricultural commodities with high mold and high moisture contents are more exposed to be contaminated with mycotoxins. Once, the agriculture product becomes infected in the field, fungi continue to propagate through the post-harvest stages as the environmental conditions are promoting fungal activity (Saad, 1991). Production of mycotoxins depends not only on fungal species, but also on many factors including substrate composition, moisture content, aeration, temperature and other storage conditions. The majority of mycotoxins of greatest 
concerns to human and animal health are mainly produced by the 4 genera of Aspergillums, Fusaria, Penicillium and Alternaria. The 4 genera so classified as "storage fungi" capable to propagate and yield mycotoxins at relative humidity of 13-18\% (Saad, 1991). Fungal species within the same genus differ in their in their requirements for optimal growth and mycotoxins synthesis. Nevertheless, hot and humid conditions still the 2 main factors affecting both fungal growth and toxin production. It's worthy to keep in mind that the presence of molds does not certainly indicate the occurrence of mycotoxins (Saad, 1991).

Kinds and types of antinutritional factors "ANF's"

\section{Enzymes "Trypsin and amylase" inhibitors}

It's well known that, comparing with agricultural commodities; legumes are proved to be the major source of ANF's. Although, protease and amylase inhibitors have been reported in many cereals, but the reported levels are quite low comparing with legumes. Within cereals, barley, rye and sorghum were proved to contain higher levels of Trypsin inhibitors could reach up to $0.45 \mathrm{gm} . / \mathrm{kg}$. Such levels of concentrations are not comparable with the corresponding concentrations of Trypsin inhibitors in soy flour which is exceeded $32 \mathrm{gm} . /$ $\mathrm{kg}$ (more than 70 folds).

\section{Haemagglutinins And Lectins}

Both phytoagglutinins and lectins are proteins in nature and are found in most of cereals and legumes. Up to the levels found in cereals, no harmful effects were reported, but considerable indications were reported when consuming less amounts of legumes might be harmful to human (Bora, 2014).

\section{Saponins}

Saponins are glycosides of high molecular weight. Legumes in general and soybean in particular, are very rich in this allellochemicals. As well, Saponins are alkaloids forming when steroidal triterpens aglycon linked to any of mono, di and tri saccharide chains, commonly occurred in wide range of consumed plants involving beans, cereals, roots, tea and medicinal herbs at very wide range $0-5-5 \% \mathrm{w} / \mathrm{w}$ of dry plant weight.

\section{Phytates}

Phytates are inositols with multi phosphate groups. Such allelochemicals are closely associated with proteins isolated in bran at high levels of concentrations could reach up to $10 \%$. Phytates contain complex of $\mathrm{Zn}$, $\mathrm{Fe}, \mathrm{Mn}$ and $\mathrm{Ca}$. So, they avoid the necessary mineral absorption, which could mineral deficiency in human and animal.

\section{Oligosaccharides and Isoflavonoids}

The ANF's of oligosaccharides and Isoflavonoids had very bioactive effect towards the processes of cancer promotion and propagation. The level of concentration of such products could reach up to $20 \%$ of the legume weight. Very recently, isoflavones were proved to inhibit tyrosine kinase, the enzyme which often stimulated in cancer cells. Thus leads to approve the hypothesis that some legumes rich in isoflavones like soybean can prevent against cancer (Bora, 2014).

\section{Non protein amino acids "NPAA"}

It's well known that all protein amino acids are characterized by certain definition as alpha amino carboxylic acids, due to the localization of the amine group at the position alpha. Otherwise, the amino acids considered to be NPAA. So far, more than 900 NPAA's are naturally occurred and identified in plants, especially abundant in legumes (Embaby, 2011). In plant tissues, NPAA's could act as nitrogen sources during seed germination. As well, they are important in plant/ plant, plant/ microbes, plant/ herbivores interactions.

\section{Phenolic compounds}

Phenolic compounds are proved to act as antioxidants to scavenge free radicals and chelators of prooxidant metals. Phenolic compounds could prevent low density lipoprotein (LDL) oxidation and DNA strand scission or in general enhancing immune functions. Phenolic compounds are occurred in a very wide range of concentrations (10 ug $-40 \mathrm{gm}) / \mathrm{kg}$, based on plant species. They fund in cereals, legumes, vegetables, fruits, tea, cocoa and medicinal herbs.

\section{Alkaloids}

Alkaloids are commonly occurred in legumes resulting the undesirable bitter taste. Alkaloids could be commonly and easily removed or eliminated by soaking for a few hours before consuming. 


\section{Cyanogens}

Cyanogens are occurred naturally as glycosides of hydroxyl nit riles. So, cyanogens could be utilized by plant tissues as mobile nitrogen storage compounds. They had bitter taste and moderate toxic effects on human and animals.

\section{Anti-vitamin factors}

Anti-vitamin factors are a family of ANF's representing nitrogenous compounds, commonly hydrophilic, capable to form complexes preventing the utilization of the water soluble vitamins, in particular.

However, it's well agreed that all the above mentioned groups of the secondary metabolites are produced in nature for urgent functions of the organisms producing them (Singh et al., 2015). ANF's can act as; 1) competitive weapons against other herbivores, 2) as minerals transporting agents, 3) agents of symbiosis between other organisms, 4) acting as sexual hormone precursors and 5) stimulators and inhibitors for sporulation and germination (Demain, 2000). ANF's simultaneously increases the reproductive performance of plants and make edible plants undesirable to other herbivores to maintain such nutritive plant food as important source for human consumption. It could be concluded that the allelochemicals of ANF's were anciently thought to be functionless or metabolic wastes and/ or natural toxicants. But recently, such secondary metabolites are reidentified as important bioactive substances. Regarding human and animal health, low levels of ANF's has positive effects or might be functionless in most cases, but in few cases some ANF's at high concentrations might cause negative effects.

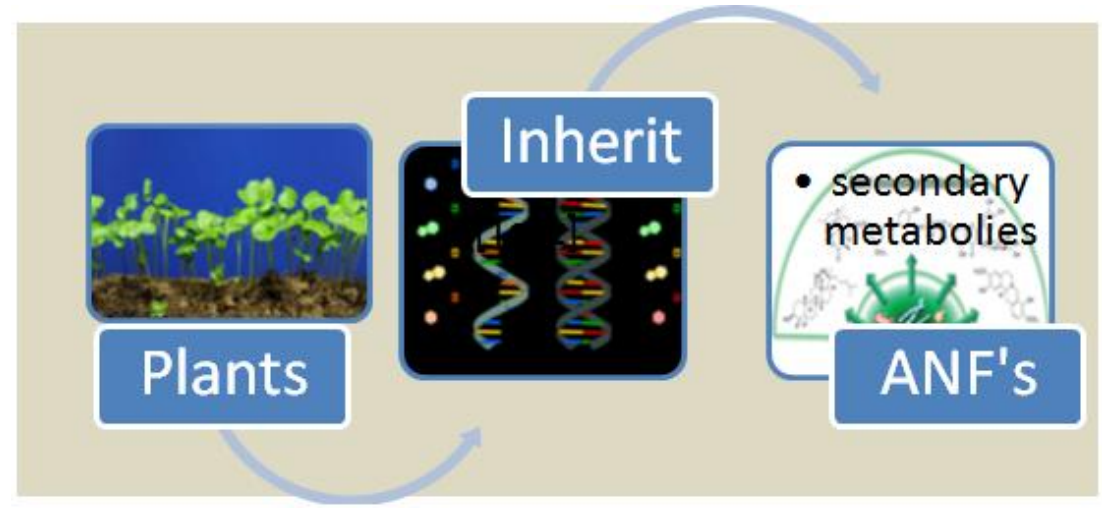

Figure. 1 Occurrence of ANF's

Mycotoxins occurrence and prevalence FAO (1979) estimated that about $25 \%$ of the cereals produced in the world were contaminated by mycotoxins, while other foodstuffs and agriculture commodities showed different degrees of mycotoxins contamination. Mycotoxins are highly toxic secondary metabolites naturally occurred and produced when certain species of fungi infected and invaded agricultural commodities and foodstuffs (Saad, 1991). Mycotoxins producing fungi commonly sub-divided into, field fungi and storage fungi. Moisture, relative humidity and temperature have the major influence on mold growth and mycotoxins(s) production. Pathogenic and toxic strains of fungi invade the pre-harvest crops require higher moisture levels $(200-250 \mathrm{~g} / \mathrm{kg})$ for infection, while invasion with storage fungi require less moisture levels $(130-180 \mathrm{~g} / \mathrm{kg})$ for the ideal proliferation and propagation (Rodrigues and Karin, 2012). Mycotoxins could occur at various points of food chain during post-harvest, especially when water activity of the stored commodities increases to levels $(13-18 \%)$ allowing the optimal conditions for fungal growth and mycotoxins(s) production. Mycotoxins could also accumulate in processed products. The reason for the production of mycotoxins is not known, so far, but all the available literature confirmed that they are not necessary even for fungal growth and development. Mycotoxins strongly resist the decomposition and breakdown processes of ingestion, besides they are proved to be thermo stable (Adejumo and Adejore, 2014). The micro fungi of the 4 genera of Aspergillum, Penicillium, Fusaria and Alternaria are recently well known as the most abundant pathogens and saprophytes of storage fungi. Many species of the 4 genera are not only capable to cause spoilage of food crops and agriculture commodities, but also had the capability to yield mycotoxins. The available mycotoxins detected and identified though to be more than 350, all are not essential for fungal growth and all had toxic effects on human and animals called "mycotoxicosis". Mycotoxicosis occurred when one or more of mycotoxins enter the body orally by ingesting contaminated food. It's worthy to differentiate between mycotoxicosis and mycosis. Mycosis is occurred when molds and fungi infect body tissues (Adejumo and Adejoro, 2014).

The most common and occurred mycotoxins 


\section{Aflatoxins}

Aflatoxins are furano-coumarin derivatives produced by some strains of Aspergillus flavus and/ or Aspergillus parasiticus. Aflatoxins are the most studied mycotoxins. Ingesting aflatoxins by human or animals leads to both acute and chronic aflatoxicosis. Acute cases result in death and chronic aflatoxicosis leads to mutagenic and carcinogenic effects besides immune suppression. Aflatoxins were previously identified in 4 types B1, B2, G1 and G2, but now more than 23 aflatoxins were identified and characterized (Hsieh, 1988).

\section{Ochratoxins}

Ochratoxin-A, the parent compound is mainly produced by Aspergillus ochraceus and some strains of Penicillium sp. The 4 types of Ochratoxins A, B, C and D were identified as nephrotoxic molecules. It's worthy to pay more attention to the possibility of Ochratoxins toxicity "ochratoxicosis" in patients with symptoms of renal pathology (Creppy, 1999).

\section{Trichothecenes}

Trichothecenes constitute a large family of more than 60 metabolite produced by fungal genera of Fusaria, Stachybotrys, Trichoderma and others. Trichothecenes as food contaminants can result alimentary hemorrhage, vomiting, while direct contact causes dermatitis. The Trichothecenes of T-2 and diacetoxyscripenol are associated with a human disease called alimentary toxic aleukia (ATA) which had certain symptoms include inflammation of the skin, vomiting and damage to hematopoietic tissues.

\section{Citrinin}

Citrinin is mainly produced by certain species of Penicillium and Aspergillus. Citrinin has been associated with yellow rice disease in Japan. Citrinin can act synergistically with another mycotoxins "Ochratoxin-A" to depress RNA synthesis.

\section{Fumonisins}

Fumonisins are produced by a number of Fusaria sp. The occurrence of Fumonisins is correlated to higher incidences of esophageal cancer in regions of South Africa, China and Italy (Peraica et al., 1999). Unlike most mycotoxins, Fumonisins are hydrophobic which make them difficult to study and evaluate.

\section{Patulin}

Patulin is produced by some strains of Penicillium. In addition to Patulin antibacterial, antiviral, antiprotozoal activity, it has toxic effects on human and animals. Patulin is regularly found in unfermented apple and other fruit juices.

\section{Zearalenone}

Zearalenone is produced by Fusaria sp., recently is classified as non-steroidal estrogen or mycoestrogen. Sometimes it's called a phytoestrogen. Zearalenol, the reduced form of Zearalenone, could increase estrogenic activity. The biological potency of Zearalenone family is high, but the actual toxicity is low (Heffron, 1999).

\section{Tremorgns}

Tremorgns are produced by certain strains of Aspergillus, Penicillium and Claviceps. The tremorgenic mycotoxins include at least 10 secondary metabolites including penitrem-A, a compound implicated in several classes of canine intoxication and some case of human tremor, vomiting and bloody diarrhea (Hocking et al., 1988). However, mycotoxins exposure could affect; digestive system (aflatoxins - T-2 toxin and vomitoxin), vascular system (Tremorgns), urinary system (Ochratoxins and Citrinin), reproductive system (Zearalenone - T2 toxin), and immune system "aflatoxins - DON" (Saad, 1991).

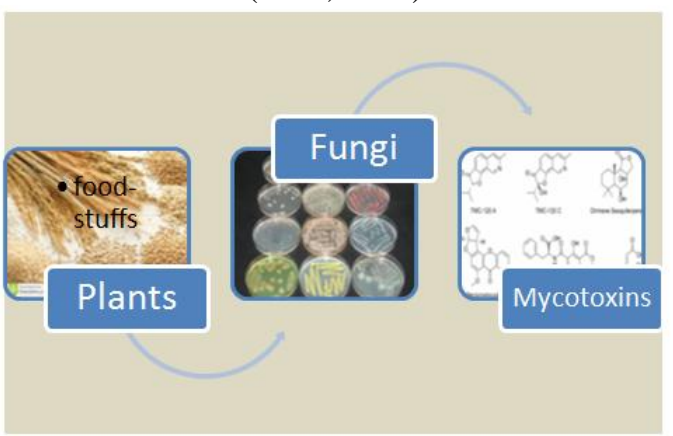

Figure 2. Occurrence of mycotoxins 


\section{Competence and difference between ANF's and Mycotoxins}

\begin{tabular}{|l|l|}
\hline ANF's & Mycotoxins \\
\hline Secondary metabolites & Secondary metabolites \\
\hline Naturally occurred & Naturally occurred \\
\hline Molecular weight: from medium to high & Molecular weight: from low to medium \\
\hline Occurred pre-harvest & Occurred post-harvest \\
\hline Moderately toxic & Highly toxic \\
\hline Physical hazards & Chemical hazards \\
\hline $\begin{array}{l}\text { Affecting utilization of nutrients, only when } \\
\text { ingested by human }\end{array}$ & Affecting human health causing Mycotoxicosis \\
\hline $\begin{array}{l}\text { Necessary to protect plant from herbivores } \\
\text { Occurred as response to genes expression }\end{array}$ & $\begin{array}{l}\text { Not yet known their benefits to plant, fungi or } \\
\text { human }\end{array}$ \\
\hline $\begin{array}{l}\text { Carry positive effects on human and animal at } \\
\text { low doses }\end{array}$ & $\begin{array}{l}\text { Had negative effects on human and animal at } \\
\text { low doses }\end{array}$ \\
\hline Not lethal & May be lethal \\
\hline $\begin{array}{l}\text { Only affecting metabolism and utilization of } \\
\text { nutrients }\end{array}$ & \begin{tabular}{l} 
Affecting certain, tissues, organs and systems \\
\hline
\end{tabular} \\
\hline
\end{tabular}

\section{References}

[1]. Adejumo, T. and Adejero, D. (2014). Incidence of aflatoxins, Fumonisins And Ochratoxins in Nigerian food. J. Food.Sci. \& Qualit.Man.Vol., 31: 127 - 148.

[2]. Bora, B. (2014). Legumes as souces of ANF's. J. of Academia and Industrial Research "JAIR", Vol., 3(6): 285-291.

[3]. Creppy, E. (1999). Human ochratoxicosis. J. Tocicol. Toxin. Rev., Vol., 18: 277 - 293.

[4]. Demain, F. (2000). The natural functions of secondary metabolites. J. Adv. Biochem. Eng. Biotech., $69: 1$ - 39.

[5]. Embaby, H. (2011). Effect of heat treatment on certain antinutrients. J. Food Sci. \& Techn., Vol., $17(1): 31-38$

[6]. FAO (1979). Perspective on mycotoxins. Food and Agriculture Organiz.,UN, Rome, ISBN: 92-5-100-870-1.

[7]. Fujii, Y. and Hiradate, S. (2007). Allelopathy - new concepts and Metyhodology. Publ. Sci. Enfield.

[8]. Gemede, H. and Ratta, N. (2014). Antinutritional factors in plant foods Potential health benefit s and adverse effects. Int. J. of Nut.Food Sci., Vol., 3(4): 284 - 289.

[9]. Heffron, J. (1999). Endocrine disruptures are they an insidious risk to Health? J. of Biochem., 1990: 28-31.

[10]. Hocking, A., Holds, K. and Tobin, N. (1988). Intoxication by tremorgenic Mycotoxins. Aus. Vet. J., Vol., 65(3): 82-85.

[11]. Hsieh, D. (1988). Potential human health hazard of mycotoxins. Pp: 69- 80 in mycotoxins and phytotoxins, Elsevier, Amsterdam.

[12]. Peracia, M., Radic, B., Lucic, A. and Favlovic, M. (1999). Toxic effects of Mycotoxins in humans. Bull. World Health Org., Vol., 77(9): 754-766.

[13]. Peter, M. Wedny, Z., Shery, F. and Benjamin, P. (2014). Alternaria toxins In grain foods in Canada. J. Mycotoxn Res., Vol., 28(4):261 -266

[14]. Rocio, C., Aurrora, L. nd Ana, L. (2007). Allelochemicals stress can trigger Oxidative damage in receptor plants. J. Plant Signaling and Behaviour, Vol., 2(4): 269 - 270.

[15]. Rodrigues,R. and Karine, A. (2014). A 3 year survey on the world wide Occurrence of mycotoxins in feedstuffs and feeds. J. of Toxins, Vol., 4: $663-675$.

[16]. Saad, M.M. (1991). Mycotoxins as agriculture, environmental and health Problems. "A Book in Arabic". Published by the Egyptian Auth. Of Books, 1991.

[17]. Singh, P., Nidhi, S., Bechan, S. and Sameer, S. (2015). Effect of domestic Processes on chickenpeas for antinutritional contents and Their divergence. Am. J. Food Sci. \& Technol. , Vol., 3(4): 111 - 117. 